\title{
Half-arc-transitive graphs of order $4 p$ of valency twice a prime
}

\author{
Xiuyun Wang, Yan-Quan Feng * \\ Department of Mathematics, Beijing Jiaotong University \\ Beijing 100044, P. R. China
}

Received 9 November 2009, accepted 1 May 2010, published online 25 October 2010

\begin{abstract}
A graph is half-arc-transitive if its automorphism group acts transitively on vertices and edges, but not on arcs. Let $p$ be a prime. Cheng and Oxley [On weakly symmetric graphs of order twice a prime, J. Combin. Theory B 42(1987) 196-211] proved that there is no half-arc-transitive graph of order $2 p$, and Alspach and Xu [ $\frac{1}{2}$-transitive graphs of order $3 p$, J. Algebraic Combin. 3(1994) 347-355] classified half-arc-transitive graphs of order 3p. In this paper we classify half-arc-transitive graphs of order $4 p$ of valency $2 q$ for each prime $q \geq 5$. It is shown that such graphs exist if and only if $p-1$ is divisible by $4 q$. Moreover, for such $p$ and $q$ a unique half-arc-transitive graph of order $4 p$ and valency $2 q$ exists and this graph is a Cayley graph.
\end{abstract}

Keywords: Cayley graph, half-arc-transitive graph, transitive graph.

Math. Subj. Class.: 05C25, 20B25

\section{Introduction}

Throughout this paper we denote by $\mathbb{Z}_{n}$ the cyclic group of order $n$ as well as the ring of residue classes modulo $n$, and by $\mathbb{Z}_{n}^{*}$ the multiplicative group of the ring $\mathbb{Z}_{n}$. Let $D_{2 n}$ be the dihedral group of order $2 n$, and let $A_{n}$ and $S_{n}$ be the alternating and symmetric group of degree $n$, respectively. All graphs are assumed to be finite, simple and undirected, but with an implicit orientation of the edges when appropriate. For a graph $X$, let $V(X)$, $E(X), A(X)$ and $\operatorname{Aut}(X)$ be the vertex set, the edge set, the arc set and the automorphism group of $X$, respectively. A graph $X$ is said to be vertex-transitive, edge-transitive or arctransitive (symmetric) if $\operatorname{Aut}(X)$ acts transitively on $V(X), E(X)$, or $A(X)$, respectively, and half-arc-transitive if $X$ is vertex-transitive and edge-transitive, but not arc-transitive.

\footnotetext{
* Corresponding author.

E-mail addresses: wxy830815@gmail.com (Xiuyun Wang), yqfeng@bjtu.edu.cn (Yan-Quan Feng)
} 
More generally, by a half-arc-transitive action of a subgroup $G$ of $\operatorname{Aut}(X)$ on a graph $X$ we shall mean a vertex-transitive and edge-transitive, but not arc-transitive action of $G$ on $X$. In this case, we shall say that the graph $X$ is $G$-half-arc-transitive.

The investigation of half-arc-transitive graphs was initiated by Tutte [34] and he proved that a vertex- and edge-transitive graph with odd valency must be arc-transitive. In 1970 Bouwer [4] constructed a $2 k$-valent half-arc-transitive graph for every $k \geq 2$ and later more such graphs were constructed (see [10, 15, 17, 18, 33]). Let $p, q$ be odd primes. It is well-known that there are no half-arc-transitive graphs of order $p$ or $p^{2}$, and by Cheng and Oxley [6], there are no half-arc-transitive graphs of order $2 p$. Alspach and Xu [2] classified half-arc-transitive graphs of order $3 p$ and Wang [35] classified half-arc-transitive graphs of order a product of two distinct primes. Despite all of these efforts, however, more classifications of half-arc-transitive graphs with general valencies seem to be very difficult. For example, classification of half-arc-transitive graphs of order $4 p$ has been considered for more than 10 years by many authors, but it has still not been completed. Recently, classifications of tetravalent and hexavalent half-arc-transitive graphs of order $4 p$ were given in [13] and [37], respectively. In fact, investigation of half-arc-transitive graphs of small valencies is currently an active topic in algebraic graph theory. For more information, see [1, 7, 11, 12, 14, 21, 22, 23, 24, 25, 26, 27, 28, 29, 30, 32, 38, 39]. In this paper we classify $2 q$-valent half-arc-transitive graphs of order $4 p$ for each prime $q \geq 5$. It is shown that such graphs are Cayley and exist if and only if $p-1$ is divisible by $4 q$. Moreover, for a given order such a graph is unique.

To end this section, we introduce the so called quotient graph of a graph $X$. Let $\Sigma=$ $\left\{B_{0}, B_{1}, \ldots, B_{n-1}\right\}$ be a partition of $V(X)$. The quotient graph $X_{\Sigma}$ of $X$ relative to the partition $\Sigma$ is defined to have vertex set and edge set as follows:

$$
\begin{aligned}
& V\left(X_{\Sigma}\right)=\Sigma \\
& E\left(X_{\Sigma}\right)=\left\{\left\{B_{i}, B_{j}\right\} \mid \text { there exist } v_{i} \in B_{i}, v_{j} \in B_{j} \text { such that }\left\{v_{i}, v_{j}\right\} \in E(X)\right\} .
\end{aligned}
$$

In particular, if $N \leq \operatorname{Aut}(X)$ then the set of orbits of $N$ on $V(X)$ is a partition of $V(X)$. In this case, the quotient graph of $X$ relative to the orbits of $N$ is also called the quotient graph of $X$ relative to $N$, denoted by $X_{N}$. It is easy to see that if $N \unlhd G \leq \operatorname{Aut}(X)$ and $G$ is transitive on edges of $X$ then the valency of $X_{N}$ is a divisor of the valency of $X$.

\section{Preliminary results}

For a finite group $G$ and a subset $S$ of $G$ such that $1 \notin S$ and $S=S^{-1}$, the Cayley graph $\operatorname{Cay}(G, S)$ on $G$ with respect to $S$ is defined to have vertex set $G$ and edge set $\{\{g, s g\} \mid g \in G, s \in S\}$. The following facts about Cayley graph are well known (see [3]). Given $g \in G$, define the permutation $R(g)$ on $G$ by $x \mapsto x g, x \in G$. Then the right regular representation $R(G)=\{R(g) \mid g \in G\}$ is a regular subgroup of $\operatorname{Aut}(\operatorname{Cay}(G, S))$, and $\operatorname{Aut}(G, S)=\left\{\alpha \in \operatorname{Aut}(G) \mid S^{\alpha}=S\right\}$ is a subgroup of the stabilizer $\operatorname{Aut}(\operatorname{Cay}(G, S))_{1}$ of the vertex 1 in $\operatorname{Aut}(\operatorname{Cay}(G, S))$. Furthermore, A graph $X$ is isomorphic to a Cayley graph on $G$ if and only if its automorphism group $\operatorname{Aut}(X)$ has a subgroup isomorphic to $G$, acting regularly on vertices.

A Cayley graph $\operatorname{Cay}(G, S)$ is said to be normal if $\operatorname{Aut}(\operatorname{Cay}(G, S))$ contains $R(G)$ as a normal subgroup. The following proposition is fundamental for normal Cayley graphs.

Proposition 2.1. [38, Proposition 1.5] Let $X=\mathrm{Cay}(G, S)$ be a Cayley graph on a finite group $G$ with respect to $S$. Let $A=\operatorname{Aut}(X)$ and let $A_{1}$ be the stabilizer of 1 in $A$. Then $X$ 
is normal if and only if $A_{1}=\operatorname{Aut}(G, S)$.

Cheng and Oxley [6] classified the connected symmetric graphs of order $2 p$ for a prime $p$. To extract a classification of connected $q-, 2 q$ - and $4 q$-valent symmetric graphs of order $2 p$ for a prime $q \geq 5$, we need to define some graphs. Let $V$ and $V^{\prime}$ be two disjoint copies of $\mathbb{Z}_{p}$, say $V=\left\{i \mid i \in \mathbb{Z}_{p}\right\}$ and $V^{\prime}=\left\{i^{\prime} \mid i \in \mathbb{Z}_{p}\right\}$. Let $r$ be a positive integer dividing $p-1$ and $H(p, r)$ the unique subgroup of $\mathbb{Z}_{p}^{*}$ of order $r$. Define the graph $G(2 p, r)$ to have vertex set $V \cup V^{\prime}$ and edge set $\left\{x y^{\prime} \mid x, y \in \mathbb{Z}_{p}, y-x \in H(p, r)\right\}$. Clearly, $G(2 p, p-1) \cong K_{p, p}-p K_{2}$, the complete bipartite graph of order $2 p$ minus a 1 -factor. Furthermore, assume that $r$ is an even integer dividing $p-1$. Then the graph $G(2, p, r)$ is defined to have vertex set $V \cup V^{\prime}$ and edge set $\left\{x y, x^{\prime} y, x y^{\prime}, x^{\prime} y^{\prime} \mid x, y \in \mathbb{Z}_{p}, y-x \in\right.$ $H(p, r)\}$. The lexicographic product $X[Y]$ of graph $X$ by graph $Y$ is the graph with vertex set $V(X[Y])=V(X) \times V(Y)$ and with two vertices $u=\left(x_{1}, y_{1}\right)$ and $v=\left(x_{2}, y_{2}\right)$ adjacent whenever $x_{1}$ is adjacent to $x_{2}$, or $x_{1}=x_{2}$ and $y_{1}$ is adjacent to $y_{2}$. Clearly, if $X$ is symmetric and $Y$ is a graph with no edge, then $X[Y]$ is symmetric. Moreover, $G(2, p, r)$ is in fact the lexicographic product of a circulant $\operatorname{Cay}\left(\mathbb{Z}_{p}, H(p, r)\right)$ by $2 K_{1}$.

Proposition 2.2. [6, Theorem 2.4 and Table 1] Let $p, q$ be odd primes with $q \geq 5$ and let $X$ be a connected edge-transitive graph of order $2 p$. Then $X$ is symmetric. Furthermore, if $X$ has valency $q$ then one of the following holds:

(1) $X \cong K_{2 p}$, the complete graph of order $2 p$, and $2 p-1=q$;

(2) $X \cong K_{p, p}$, the complete bipartite graph of order $2 p$, and $p=q$;

(3) $X \cong G(2 p, q)$ with $q \mid(p-1)$ and $(p, q) \neq(11,5)$, and $\operatorname{Aut}(X) \cong\left(\mathbb{Z}_{p} \rtimes \mathbb{Z}_{q}\right) \rtimes \mathbb{Z}_{2}$;

(4) $X \cong G(2 \cdot 11,5)$ and $\operatorname{Aut}(X) \cong \operatorname{PSL}(2,11) \rtimes \mathbb{Z}_{2}$.

If $X$ has valency $2 q$ then $X$ is bipartite and one of the following holds:

(5) For $2 q<p-1, X \cong G(2 p, 2 q)$ with $2 q \mid(p-1)$ and $\operatorname{Aut}(X) \cong\left(\mathbb{Z}_{p} \rtimes \mathbb{Z}_{2 q}\right) \rtimes \mathbb{Z}_{2}$;

(6) For $2 q=p-1, X \cong K_{p, p}-p K_{2}$ and $\operatorname{Aut}(X) \cong S_{p} \rtimes \mathbb{Z}_{2}$.

If $X$ has valency $4 q$ then one of the following holds:

(7) $X$ is non-bipartite, $X \cong G(2, p, 2 q)$ with $2 q \mid p-1$; for $2 q<p-1$, $\operatorname{Aut}(X) \cong$ $\mathbb{Z}_{2}^{p} \rtimes\left(\mathbb{Z}_{p} \rtimes \mathbb{Z}_{2 q}\right)$ and for $2 q=p-1$, Aut $(X) \cong \mathbb{Z}_{2}^{p} \rtimes S_{p} ;$

(8) $X$ is bipartite and $X \cong G(2 p, 4 q)$ with $4 q \mid(p-1)$; for $4 q<p-1$, $\operatorname{Aut}(X) \cong$ $\left(\mathbb{Z}_{p} \rtimes \mathbb{Z}_{4 q}\right) \rtimes \mathbb{Z}_{2}$ and for $4 q=p-1, X \cong K_{p, p}-p K_{2}$ and $\operatorname{Aut}(X) \cong S_{p} \rtimes \mathbb{Z}_{2}$.

Let $G$ act transitively on a set $\Omega$. Then $G$ induces a natural action on $\Omega \times \Omega$ defined by $(x, y)^{g}=\left(x^{g}, y^{g}\right)$ for $(x, y) \in \Omega \times \Omega$ and $g \in G$. The orbits of $G$ on $\Omega \times \Omega$ are called orbitals of $G$. The orbital $\Delta=\{(x, x) \mid x \in \Omega\}$ of $G$ is trivial and all other orbitals of $G$ are nontrivial. Let $O$ be a nontrivial orbital of $G$. The pair $(\Omega, O)$, denoted by $\mathcal{O}$, is a directed graph with vertex set $\Omega$ and directed edge set $O$, called the orbital digraph of $G$ relative to $O$. For any orbital $O$ of $G$, it is easy to show that $O^{*}=\{(\alpha, \beta) \mid(\beta, \alpha) \in O\}$ is also an orbital of $G$, called the paired orbital of $O$, and $O$ is said to be self-paired if $O^{*}=O$. Clearly, if $O$ is a non-self-paired orbital then the underlying graph of $\mathcal{O}$ is $G$ half-arc-transitive. Conversely, if $X$ is a half-arc-transitive graph then $X$ is the underlying graph of an orbital digraph $(V(X), O)$ of $\operatorname{Aut}(X)$ relative to a non-self-paired orbital $O$. In this case, $\operatorname{Aut}(X)$ coincides with the automorphism group of the digraph $(V(X), O)$. This implies the following proposition. 
Proposition 2.3. Let $X$ be a connected half-arc-transitive graph of valency $2 n$. Let $A=$ $\operatorname{Aut}(X)$ and let $A_{u}$ be the stabilizer of $u \in V(X)$ in $A$. Then each prime divisor of $\left|A_{u}\right|$ is a divisor of $n$ !.

By [10, Lemma 2.2], we have the following proposition (also see [1, 19, 31, 36]).

Proposition 2.4. The smallest half-arc-transitive graph has order 27. The smallest vertexprimitive half-arc-transitive graph of order $k p$, with $p$ a prime and $k<p$, has order 253.

The following proposition is straightforward (also see [13, Propositions 2.1 and 2.2]).

Proposition 2.5. Let $X=\operatorname{Cay}(G, S)$ be a half-arc-transitive graph. Then, there is no involution in $S$, and no $\alpha \in \operatorname{Aut}(G, S)$ such that $s^{\alpha}=s^{-1}$ for some $s \in S$. In particular, there are no half-arc-transitive Cayley graphs on abelian groups.

Let $G$ be a transitive permutation group on a set $\Omega$. A nonempty subset $\Delta$ of $\Omega$ is called a block for $G$ if for each $g \in G$, either $\Delta^{g}=\Delta$ or $\Delta^{g} \cap \Delta=\phi$. Clearly, the set $\Omega$, the empty set $\phi$, and the sets $\{\alpha\}$ consisting of only one point are blocks of $G$ on $\Omega$. We call these trivial blocks. A transitive group $G$ is said to be primitive if $G$ has only trivial blocks, and imprimitive if there is at least one non-trivial block. The socle of a finite group $G$, denoted by $\operatorname{soc}(G)$, is the product of all minimal normal subgroups of $G$. One may extract the following results from [20, Table 3].

Proposition 2.6. Let $p$ be a prime and $G$ a primitive group of degree $n$.

(1) For $n=p$, G is either solvable with a normal Sylow p-subgroup or non-solvable with the following table, where $d$ and $k$ denote the degree and transitive multiplicity, respectively.

\begin{tabular}{llll}
\hline $\operatorname{soc}(\mathrm{G})$ & $\mathrm{d}$ & $\mathrm{k}$ & comment \\
\hline$A_{p}$ & $p$ & $p-2$ & $G=A_{p}$ \\
$A_{p}$ & $p$ & $p$ & $G=S_{p}$ \\
$\operatorname{PSL}\left(2,2^{2^{s}}\right)$ & $p=2^{2^{s}}+1$ & 3 & $s>0$ \\
$\operatorname{PSL}(n, q)$ & $p=\frac{q^{n}-1}{q-1}$ & 2 & $n \geq 3, n$ odd \\
$\operatorname{PSL}(2,11)$ & 11 & 2 & \\
$M_{11}$ & 11 & 4 & \\
$M_{23}$ & 23 & 4 & \\
\hline
\end{tabular}

(2) For $n=2 p$, either $G$ is 2-transitive or $p=5$.

(3) For $n=4 p$, either $G$ is 2-transitive or $p=7,13$, or 17 .

One may deduce Proposition 2.6(1) and 2.6(2) also from [9, Corollary 3.5B] and [6, Theorem 1.1], respectively. Moreover, if $G$ is primitive, but not 2-transitive of degree $2 p$ then $p=5$ and $G \cong A_{5}$ or $S_{5}$. If $G$ is primitive, but not 2-transitive of degree $4 p$ then $G \cong A_{8}, S_{8}, \operatorname{PSL}(2,8)$ or $\operatorname{PGL}(2,7)$ for $p=7, G \cong \operatorname{Aut}(\operatorname{PSL}(3,3))$ for $p=13$, or $G$ is isomorphic to a subgroup between $\operatorname{PSL}(2,16)$ and $\operatorname{P\Gamma L}(2,16)$ for $p=17$. 


\section{Main result}

Let $p, q$ be odd primes with $q \geq 5$. In this section we classify the $2 q$-valent half-arctransitive graphs of order $4 p$. Following the notation of Kwak et al. [16], for any integer $\ell$ and any positive integer $t$, define

$$
\ell[t]=\ell^{t-1}+\ell^{t-2}+\cdots+1 .
$$

Let $G=\left\langle a, b \mid a^{p}=b^{4}=1, b^{-1} a b=a^{r}\right\rangle$, where $r^{2} \equiv-1(\bmod p)$. It is easy to see that $r$ is an element of order 4 in $\mathbb{Z}_{p}^{*}$ and the group $G$ is independent of the choice of $r$. In particular, $p-1$ is divisible by 4 . Furthermore, assume that $p-1$ is divisible by $q$. Then there is a unique subgroup of order $q$, say $\langle s\rangle$, in $\mathbb{Z}_{p}^{*}$. Set $T=\left\{b, a^{s[1]} b, a^{s[2]} b, a^{s[3]} b, \ldots, a^{s[q-1]} b\right\}$ and $S=T \cup T^{-1}$. Define

$$
\mathcal{C}_{4 p}^{2 q}=\operatorname{Cay}(G, S)
$$

Clearly, $s[i] \neq 0(\bmod p)$ for each $1 \leq i \leq q-1$, that is $s[i] \in \mathbb{Z}_{p}^{*}$, and one may easily show that $\mathcal{C}_{4 p}^{2 q}$ is a connected graph of order $4 p$ and of valency $2 q$.

Clearly, $s^{i}(1 \leq i \leq q-1)$ are all the elements of order $q$ in $\mathbb{Z}_{p}^{*}$. Set $S_{i}=T_{i} \cup T_{i}^{-1}$ with $T_{i}=\left\{b, a^{s^{i}[1]} b, a^{s^{i}[2]} b, a^{s^{i}[3]} b, \ldots, a^{s^{i}[q-1]} b\right\}$. Then $T_{1}=T$ and $S_{1}=S$. For any given $1 \leq i \leq q-1$, the map $a \mapsto a^{s[i]^{-1}}, b \mapsto b$, induces an automorphism of $G$, say $\beta_{i}$. For each $1 \leq j \leq q-1$, there is $1 \leq k \leq q-1$ such that $s^{j}=\left(s^{i}\right)^{k}$. It follows that

$$
s[j] s[i]^{-1}=\frac{1-s^{j}}{1-s} \frac{1-s}{1-s^{i}}=\frac{1-\left(s^{i}\right)^{k}}{1-s^{i}}=s^{i}[k],
$$

which means $T_{1}^{\beta_{i}}=T_{i}$. Thus, $\beta_{i}$ is an isomorphism from $\operatorname{Cay}(G, S)$ to $\operatorname{Cay}\left(G, S_{i}\right)$. Therefore, $\mathcal{C}_{4 p}^{2 q}$ is independent of the choice of the element $s$ of order $q$ in $\mathbb{Z}_{p}^{*}$.

Denote by $\alpha$ the automorphism of $G$ induced by $a \mapsto a^{s}, b \mapsto a b$. Note that $s[q]=$ $s^{q-1}+s^{q-2}+\cdots+s+1=0(\bmod p)$. Then $b^{\alpha}=a b,\left(a^{s[i]} b\right)^{\alpha}=a^{s[i+1]} b$ for each $1 \leq i \leq q-2$, and $\left(a^{s[q-1]} b\right)^{\alpha}=b$. It follows that $\alpha \in \operatorname{Aut}(G, S)$, implying that $\operatorname{Aut}\left(\mathcal{C}_{4 p}^{2 q}\right)$ has an edge-transitive subgroup of order $4 p q$, that is $R(G) \rtimes\langle\alpha\rangle$. It will be shown in Lemma 3.2 that $\operatorname{Aut}\left(\mathcal{C}_{4 p}^{2 q}\right)=R(G) \rtimes\langle\alpha\rangle$ and hence $\mathcal{C}_{4 p}^{2 q}$ is half-arc-transitive. The following is the main result of this paper.

Theorem 3.1. Let $p, q$ be odd primes with $q \geq 5$. Then $X$ is a $2 q$-valent half-arc-transitive graph of order $4 p$ if and only if $4 q \mid(p-1)$ and $X \cong \mathcal{C}_{4 p}^{2 q}$ with $\operatorname{Aut}\left(\mathcal{C}_{4 p}^{2 q}\right)=R(G) \rtimes\langle\alpha\rangle$.

The sufficiency is proved as a part of Lemma 3.2 and the necessity is proved in Lemma 3.3. For a graph $X$ and $u \in V(X)$, let $N_{d}(u)$ denote the set of vertices having distance $d$ from $u$ in $X$. If $X$ is a directed graph, let $N^{+}(u)$ denote the set of out-neighbors of $u$ and $N^{-}(u)$ the set of in-neighbors of $u$.

Lemma 3.2. Let $p, q$ be odd primes with $q \geq 5$. Let $X=\operatorname{Cay}(G, S)$ be a $2 q$-valent half-arc-transitive Cayley graph on a group $G$ of order $4 p$. Then $G \cong\langle a, b| a^{p}=$ $\left.b^{4}=1, b^{-1} a b=a^{r}\right\rangle$ with $r^{2}=-1(\bmod p)$. Moreover, for each prime $p$ satisfying $4 q \mid(p-1)$, the Cayley graph $\mathcal{C}_{4 p}^{2 q}$ (defined in Eq(3.1)) is half-arc-transitive and $\operatorname{Aut}\left(\mathcal{C}_{4 p}^{2 q}\right)=$ $R(G) \rtimes\langle\alpha\rangle$. 
Proof. Since there are no half-arc-transitive graphs of order $p$ or $2 p$ (see [5, 6]), $X$ is connected. Thus, $|S|=2 q, S^{-1}=S$ and $\langle S\rangle=G$. By Proposition 2.5, $G$ is non-abelian and by Proposition 2.4, $p \geq 7$. From elementary group theory, all non-abelian groups of order $4 p$ for every odd prime $p \geq 7$, up to isomorphism, can be written as follows:

$$
\begin{aligned}
& G_{1}(p)=\left\langle a, b \mid a^{2 p}=b^{2}=1, b^{-1} a b=a^{-1}\right\rangle \\
& G_{2}(p)=\left\langle a, b \mid a^{2 p}=1, b^{2}=a^{p}, b^{-1} a b=a^{-1}\right\rangle \\
& G_{3}(p)=\left\langle a, b \mid a^{p}=b^{4}=1, b^{-1} a b=a^{r}\right\rangle, r^{2} \equiv-1(\bmod p) .
\end{aligned}
$$

Clearly, $G_{3}(p)$ exists if and only if $p \equiv 1(\bmod 4)$, and $G_{1}(p)$ is the dihedral group $D_{4 p}$. By Proposition 2.5, there is no involution in $S$, and since $G=\langle S\rangle$, one has $G \neq G_{1}(p)$. Suppose $G=G_{2}(p)$. Then $S$ contains at least one element of order 4 and its inverse. Each element of order 4 is of the form $a^{i} b$ or $a^{i} b^{-1}$. The automorphism of $G_{2}(p)$ induced by $b \mapsto b^{-1}, a \mapsto a$, maps $a^{j} b$ to $\left(a^{j} b\right)^{-1}$ for any integer $j$ and fixes $\langle a\rangle$ pointwise. This is impossible by Proposition 2.5. Thus, $G=G_{3}(p)$ and $4 \mid p-1$.

Let $4 q \mid(p-1)$, and let $s$ be an element of order $q$ in $\mathbb{Z}_{p}^{*}$. Recall that $\mathcal{C}_{4 p}^{2 q}=\operatorname{Cay}(G, S)$ and $R(G) \rtimes\langle\alpha\rangle \leq \operatorname{Aut}\left(\mathcal{C}_{4 p}^{2 q}\right)$, where $S=T \cup T^{-1}$ with $T=\left\{b, a^{s[1]} b, a^{s[2]} b, a^{s[3]} b, \ldots\right.$, $\left.a^{s[q-1]} b\right\}$, and $\alpha$ is the automorphism of $G$ of order $q$ induced by $a \mapsto a^{s}, b \mapsto a b$. To finish the proof, it suffices to show that $\operatorname{Aut}\left(\mathcal{C}_{4 p}^{2 q}\right)=R(G) \rtimes\langle\alpha\rangle$.

Let $G^{*}=\left\langle a, b^{2}\right\rangle$. Then $G^{*}$ is isomorphic to $D_{2 p}$. It is easy to see that $\mathcal{C}_{4 p}^{2 q}$ is bipartite with partite sets $U=G^{*}$ and $U^{\prime}=b G^{*}$. Set $X=\mathcal{C}_{4 p}^{2 q}$ and $A=\operatorname{Aut}(X)$. Let $A^{*}$ be the subgroup of $A$ fixing the partite sets $U$ and $U^{\prime}$ of $X$ setwise. Then $A^{*}$ is transitive on both $U$ and $U^{\prime}$ and $\left|A: A^{*}\right|=2$, implying $A^{*} \triangleleft A$. Since $R(G) \leq A$, we have $A=\left\langle A^{*}, R(b)\right\rangle$. Set $R\left(G^{*}\right)=\left\{R(g) \mid g \in G^{*}\right\}$. Then $R\left(G^{*}\right) \leq R(G)$, and $R\left(G^{*}\right)$ fixes $U$ and $U^{\prime}$ setwise. Hence, $R\left(G^{*}\right) \leq A^{*}$. Since $X$ has valency $2 q$ and $4 q \mid(p-1)$, one has $|A|=4 p q n$, where each prime divisor of $n$ is less than $p$.

Claim 1: $A$ is solvable.

Suppose that $A^{*}$ is 2 -transitive in its action on $U$. Then $U \backslash\{1\}=N_{2}(1)$, the set of vertices having distance 2 from 1 . Every vertex in $U \backslash\{1\}$ has the same number of neighbors in $N_{1}(1)$, say $k$. Then $(2 p-1) k=2 q(2 q-1)$, and since $(2 p-1,2 q)=1$, one has $k=2 q$, implying $p=q$, contrary to the fact that $4 q \mid p-1$. Since $q \geq 5$ and $4 q \mid p-1$, we have $p \geq 29$, and by Proposition 2.6, $A^{*}$ is imprimitive on $U$.

Let $B$ be a non-trivial block of $A^{*}$ on $U$ containing 1 . Then $B$ is also a block of $R\left(G^{*}\right)$, forcing that $B$ is a subgroup of $G^{*}$. Thus, $\Sigma_{1}=\left\{B g \mid g \in G^{*}\right\}$ is a complete block system of $A^{*}$ on $U$. Furthermore, $\Sigma_{2}=\Sigma_{1}^{R(b)}=\left\{B g \mid g \in b G^{*}\right\}$ is a complete block system of $A^{*}$ on $U^{\prime}$. Recall that $A=\left\langle A^{*}, R(b)\right\rangle$. It follows that $\Sigma=\Sigma_{1} \cup \Sigma_{2}=\{B g \mid g \in G\}$ is a complete block system of $A$ on $V(X)$. Then the quotient graph $X_{\Sigma}$ is bipartite and the edge-transitivity of $X$ implies that $X_{\Sigma}$ is edge-transitive. Let $K$ be the kernel of $A$ acting on $\Sigma$. Since $|U|=2 p$, one has $|B|=p$ or 2 .

Assume $|B|=p$. Then $|\Sigma|=4$ and since $X_{\Sigma}$ is bipartite, $X_{\Sigma}$ is a 4-cycle, say $X_{\Sigma}=$ $\left(B_{0}, B_{1}, B_{2}, B_{3}\right)$ with $B_{0}=B$. The induced subgraph $\left\langle B_{i}, B_{i+1}\right\rangle$ of $B_{i} \cup B_{i+1}$ in $X$ has order $2 p$ and valency $q$, which cannot be isomorphic to $K_{p, p}$ because $4 q \mid(p-1)$. Since $p \geq 29$, Proposition 2.2 implies that $\left\langle B_{i}, B_{i+1}\right\rangle \cong G(2 p, q)$ and $\left|\operatorname{Aut}\left(\left\langle B_{i} \cup B_{i+1}\right\rangle\right)\right|=2 p q$. Any Sylow $p$-subgroup of $A$ is a subgroup of $K$, and since $\left|B_{i}\right|=p, K$ is primitive on $B_{i}$. Suppose that $K$ is unfaithful on $B_{i}$. Then the kernel of $K$ on $B_{i}$ is transitive on $B_{i+1}$, forcing $\left\langle B_{i}, B_{i+1}\right\rangle \cong K_{p, p}$, a contradiction. Thus, $K$ acts faithfully on $B_{i}$, implying that 
$|K|$ is a divisor of $\left|\operatorname{Aut}\left(\left\langle B_{i} \cup B_{i+1}\right\rangle\right)\right|=2 p q$. This means that $K$ is solvable. Also, $A / K$ is solvable because $A / K \leq \operatorname{Aut}\left(X_{\Sigma}\right) \cong D_{8}$. It follows that $A$ is solvable.

Assume $|B|=2$. Then $K$ is a 2-group and hence solvable. By Proposition 2.2, $X_{\Sigma}$ is a connected symmetric graph of order $2 p$. Let $X_{\Sigma}$ be of valency $d$ and let $k$ be the number of edges between $B$ and $B b$ in $X$ (note that $B$ is indeed adjacent to $B b$ in $X_{\Sigma}$ ). Clearly, $k \leq 4$ and $2 \cdot 2 q=k \cdot d$. Since $q \geq 5, k \neq 3$ and $d=q, 2 q$, or $4 q$. Recall that $4 q \mid(p-1)$ and $p \geq 29$. If $4 q<p-1$, or $4 q=p-1$ and $X_{\Sigma}$ has valency $q$ or $2 q$ then, by Proposition 2.2, $X_{\Sigma} \cong G(2 p, q), G(2 p, 2 q)$ or $G(2 p, 4 q)$ and $\operatorname{Aut}\left(X_{\Sigma}\right) \cong\left(\mathbb{Z}_{p} \rtimes \mathbb{Z}_{q}\right) \rtimes \mathbb{Z}_{2},\left(\mathbb{Z}_{p} \rtimes \mathbb{Z}_{2 q}\right) \rtimes \mathbb{Z}_{2}$ or $\left(\mathbb{Z}_{p} \rtimes \mathbb{Z}_{4 q}\right) \rtimes \mathbb{Z}_{2}$. In all these cases, $\operatorname{Aut}\left(X_{\Sigma}\right)$ is solvable, and since $A / K \leq \operatorname{Aut}\left(X_{\Sigma}\right)$, $A / K$ is solvable. Thus, $A$ is solvable. Now one may assume $4 q=p-1$ and $X_{\Sigma}$ has valency $4 q$. In this case, $X_{\Sigma} \cong K_{p, p}-p K_{2}$ and there is exactly one edge in $X$ between $B$ and $B b$, which forces that $K=1$. It follows that $A^{*}$ is faithful on $\Sigma_{1}$. Since $\left|\Sigma_{1}\right|=p$, by Proposition 2.6 either $\operatorname{soc}\left(A^{*}\right)$ is non-solvable and 2-transitive on $\Sigma_{1}$ or $A^{*}$ is solvable.

Suppose that $\operatorname{soc}\left(A^{*}\right)$ is non-solvable and 2-transitive on $\Sigma_{1}$. If $\operatorname{soc}\left(A^{*}\right)$ is not 3transitive on $\Sigma_{1}$ then, by Proposition 2.6, $\operatorname{soc}\left(A^{*}\right) \cong \operatorname{PSL}(m, r)$ because $p \geq 29$. In this case, $m \geq 3$ is an odd number, $r$ is a prime-power and $p=1+r+r^{2}+\cdots+r^{m-1}$. Since $m$ is odd, $r(1+r) \mid(p-1)$, which is impossible because $p-1=4 q$ and $q \geq 5$. Thus, $\operatorname{soc}\left(A^{*}\right)$ is 3-transitive and hence $A^{*}$ is 3-transitive on $\Sigma_{1}$. Since $X_{\Sigma} \cong K_{p, p}-p K_{2}$, one may let $\Sigma_{1}=\left\{B_{i} \mid i \in \mathbb{Z}_{p}\right\}$ and $\Sigma_{2}=\left\{B_{i}^{\prime} \mid i \in \mathbb{Z}_{p}\right\}$ such that for $k, \ell \in \mathbb{Z}_{p}, B_{k}$ is adjacent to $B_{\ell}^{\prime}$ in $X_{\Sigma}$ if and only if $k \neq \ell$. Note that for $i, j \in \mathbb{Z}_{p}$ and $i \neq j$, there is exactly one edge in $X$ between $B_{i}$ and $B_{j}^{\prime}$. This implies that for any $\alpha \in A$, we have: if $\alpha$ fixes $B_{i}$ and $B_{j}^{\prime}$ setwise then it fixes every vertex in $B_{i}$ and $B_{j}^{\prime}$ because $\left|B_{i}\right|=\left|B_{j}^{\prime}\right|=2$. Let $H$ be the subgroup of $A^{*}$ fixing $B_{0}$ and $B_{1}$. Clearly, $H$ fixes $B_{0}^{\prime}$ and $B_{1}^{\prime}$, and hence $H$ fixes every vertex in $B_{0} \cup B_{1} \cup B_{0}^{\prime} \cup B_{1}^{\prime}$. By the 3 -transitivity of $A^{*}$ on $\Sigma_{1}, H$ is transitive on $\Sigma_{1} \backslash\left\{B_{0}, B_{1}\right\}$, forcing that $X$ has valency $p-1=4 q$, contrary to the fact that $X$ has valency $2 q$. Thus, $A^{*}$ is solvable, and since $\left|A: A^{*}\right|=2, A$ is solvable. This completes the proof of Claim 1.

Let $P$ be a Sylow $p$-subgroup of $A$. Then $|P|=p$ because $|A|=4 p q n$ with each prime divisor of $n$ less than $p$.

\section{Claim 2: $P \unlhd A$.}

Let $N$ be a minimal normal subgroup of $A$. By Claim 1, $N$ is elementary abelian, and since $|V(X)|=4 p, N$ is a $p$-group or a 2-group. If $N$ is a $p$-group then $P=N \unlhd A$. Thus, one may assume that $N \cong \mathbb{Z}_{2}^{r}$ for some positive integer $r$. Since $q \geq 5$, the quotient graph $X_{N}$ of $X$ relative to $N$ has valency $q$ or $2 q$. Let $H$ be the kernel of $A$ acting on $V\left(X_{N}\right)$. Note that $X_{N}$ is edge-transitive and the orbits of $N$ are of length 2 or 4 .

Suppose first that the orbits of $N$ have length 2. Then $H$ is a 2-group and $\left|X_{N}\right|=2 p$. If $X_{N}$ has valency $q$, by Proposition $2.2, X_{N} \cong G(2 p, q)$ is a $q$-valent symmetric graph of order $2 p$ and $A / H \leq \operatorname{Aut}(G(2 p, q))=\left(\mathbb{Z}_{p} \rtimes \mathbb{Z}_{q}\right) \rtimes \mathbb{Z}_{2}$. Note that $G=G_{3}(p)$ and $G$ has no non-trivial normal 2-subgroup. This implies that $R(G) \cap H=1$ and $R(G)=$ $R(G) /(R(G) \cap H) \cong R(G) H / H \leq A / H$. Since 4||$R(G) \mid$, one has 4|| $\operatorname{Aut}(G(2 p, q)) \mid$, a contradiction. Thus, $X_{N}$ has valency $2 q$. In this case, $H_{1}=1$ and $H=N \cong \mathbb{Z}_{2}$. By Proposition $2.2, X_{N} \cong G(2 p, 2 q)$ and $\operatorname{Aut}\left(X_{N}\right)$ has a normal Sylow $p$-subgroup. It follows that $P H / H \unlhd A / H$, that is $P H \unlhd A$. Since $|H|=2, P$ is characteristic in $P H$ and hence $P \unlhd A$.

Suppose now that the orbits of $N$ have length 4 . Then $\left|X_{N}\right|=p$ and $X_{N}$ cannot have valency $q$. Thus, $X_{N}$ has valency $2 q$. In this case, $H_{1}=1$ and $H=N \cong \mathbb{Z}_{2}^{2}$. Since 
$4 q \mid(p-1), X_{N}$ cannot be a complete graph, implying that $A / H$ cannot be 2 -transitive on $V\left(X_{N}\right)$. By Proposition 2.6, $A / H$ has a normal Sylow $p$-subgroup, that is $P H / H \unlhd A / H$. Since $|H|=4, P$ is characteristic in $P H$ and hence $P \unlhd A$. This completes the proof of Claim 2.

Now we are ready to finish the proof by showing $\operatorname{Aut}\left(\mathcal{C}_{4 p}^{2 q}\right)=R(G) \rtimes\langle\alpha\rangle$. By Claim 2, $P \unlhd A$. Since $X$ is bipartite, the quotient graph $X_{P}$ of $X$ relative to $P$ is a 4 -cycle, say $X_{P}=\left(O_{0}, O_{1}, O_{2}, O_{3}\right)$. Recall that $\alpha$ is the automorphism of $G$ induced by $a \mapsto a^{s}$ and $b \mapsto a b$. Since $\alpha$ has order $q, \alpha$ fixes $O_{i}$ setwise for each $i \in \mathbb{Z}_{4}$. The induced subgraph $\left\langle O_{i}, O_{i+1}\right\rangle$ of $O_{i} \cup O_{i+1}$ in $X, i \in \mathbb{Z}_{4}$, is a $q$-valent edge-transitive graph of order $2 p$. By Proposition 2.2, $\left\langle O_{i}, O_{i+1}\right\rangle \cong G(2 p, q)$ and $\operatorname{Aut}(G(2 p, q)) \cong\left(\mathbb{Z}_{p} \rtimes \mathbb{Z}_{q}\right) \rtimes \mathbb{Z}_{2}$. Let $L$ be the kernel of $A$ acting on $V\left(X_{P}\right)$. Then $P \leq L$ and $\alpha \in L$, implying that $p q|| L \mid$. Since $\left|O_{i}\right|=p\left(i \in \mathbb{Z}_{4}\right), L$ is primitive on $O_{i}$. Suppose that $L$ is unfaithful on $O_{i}$. Then the kernel of $L$ on $O_{i}$ is transitive on $O_{i+1}$ because $L$ is primitive on $O_{i+1}$, which implies that the induced subgraph $\left\langle O_{i}, O_{i+1}\right\rangle$ is isomorphic to $K_{p, p}$. It follows that $2 p=2 q$, contrary to the fact that $4 q \mid(p-1)$. Thus, $L$ acts faithfully on $O_{i}$ and $L \lesssim \operatorname{Aut}(G(2 p, q))$, implying $|L| \mid 2 p q$. It follows that $|L|=p q$ because $L$ is intransitive on $O_{i} \cup O_{i+1}$. Note that $A / L \leq \operatorname{Aut}\left(X_{P}\right) \cong D_{8}$. Since $R(G) \rtimes\langle\alpha\rangle \leq A$, one has $|A|=4 p q$ or $8 p q$.

Suppose $|A|=8 p q$. Then $A / L=\operatorname{Aut}\left(X_{P}\right) \cong D_{8}$ and consequently $X$ is symmetric. Furthermore, $R(G) \rtimes\langle\alpha\rangle \unlhd A$ and $\left|A_{1}\right|=2 q$. Noting that $R(G)$ is characteristic in $R(G) \rtimes\langle\alpha\rangle$, we have $R(G) \unlhd A$. By Proposition 2.1, $A_{1}=\operatorname{Aut}(G, S)$ and hence $\operatorname{Aut}(G, S)=\langle\alpha, \beta\rangle$, where $\beta$ is an involution in $\operatorname{Aut}(G, S)$. Recall that $T=$ $\left\{b, a^{s[1]} b, a^{s[2]} b, a^{s[3]} b, \ldots, a^{s[q-1]} b\right\}, S=T \cup T^{-1}, X=\operatorname{Cay}(G, S)$, and $\alpha$ permutes the elements of $T$ cyclically. The arc-transitivity of $A$ implies that $\beta$ interchanges $T$ and $T^{-1}$. Thus, there is an $i$ such that $b^{\beta \alpha^{i}}=b^{-1}$, and since $\langle a\rangle$ is characteristic in $G, a^{\beta \alpha^{i}}=a^{t}$ for some $t \in \mathbb{Z}_{p}^{*}$. Note that $G$ has an automorphism mapping $a^{t}$ to $a$ and $b$ to $b$. It follows that $G$ has an automorphism $\gamma$ such that $a^{\gamma}=a$ and $b^{\gamma}=b^{-1}$. Since $b^{-1} a b=a^{r}$, one has $b^{-1} a b=b a b^{-1}$, that is $b^{2} a=a b^{2}$, a contradiction. Thus, $|A|=4 p q$ and hence $A=R(G) \rtimes\langle\alpha\rangle$, as required.

To finish the proof of Theorem 3.1, we only need to prove the following lemma.

Lemma 3.3. Let $p, q$ be odd primes with $q \geq 5$ and let $X$ be a $2 q$-valent half-arc-transitive graph of order $4 p$. Then $4 q \mid(p-1)$ and $X \cong \mathcal{C}_{4 p}^{2 q}$.

Proof. Since $q \geq 5, X$ has valency at least 10, and since there are no half-arc-transitive graphs of order $p$ or $2 p$ (see [5,6]), $X$ is connected. Let $A=\operatorname{Aut}(X)$. Recall that $X$ is an underlying graph of an orbital digraph $\mathcal{O}:=(V(X), O)$ of $A$ for some non-self-paired orbital $O$. Thus, $A=\operatorname{Aut}(\mathcal{O})$ and $\mathcal{O}$ is a directed graph with out- and in-valency equal to $q$. Furthermore, $A$ is transitive on the directed edges of $\mathcal{O}$. Since $V(X)=V(\mathcal{O})$ and $A=\operatorname{Aut}(\mathcal{O})$, in what follows we change the graph $X$ to $\mathcal{O}$ when it is necessary. Let $u \in V(X)$ and denote by $A_{u}$ the stabilizer of $u$ in $A$. Since $\left|N^{+}(u)\right|=\left|N^{-}(u)\right|=q$ is a prime, $A_{u}$ acts on $N^{+}(u)$ and $N^{-}(u)$ primitively, and there exits $\alpha \in A_{u}$ such that $\alpha$ has order $q$ and permutes the elements in $N^{+}(u)$ cyclically. This implies that $4 p q|| A \mid$, and by Proposition 2.4, $p \geq 7$. By Proposition 2.3, $|A|=4 p q n$, where each prime divisor of $n$ is a divisor of $q$ !. Let $P$ be a Sylow $p$-subgroup of $A$.

Assume that $A$ has a non-trivial normal 2-subgroup, say $N$. Then the orbits of $N$ have length 2 or 4 and since $q \geq 5$, the quotient graph $X_{N}$ of $X$ relative to $N$ has valency $q$ or $2 q$. Let $L$ be the kernel of $A$ acting on $V\left(X_{N}\right)$. Then $L \unlhd A$ and $N \leq L$. Since 
$|V(X)|=4 p$ and $N$ is a 2-group, by Propostion 2.2, $X_{N}$ is a symmetric graph of order $p$ or $2 p$.

Suppose first that the orbits of $N$ have length 4 . Then $\left|X_{N}\right|=p, X_{N}$ cannot have valency $q$. Thus, $X_{N}$ has valency $2 q$ and the stabilizer $L_{u}$ of $u$ in $L$ fixes the neighborhood of $u$ in $X$ pointwise because $X$ and $X_{N}$ have the same valency. Thus, $L_{u}=1$ and $L$ acts regularly on each orbit of $N$, forcing $|L|=4$. It follows that $P L$ is regular on $V(X)$ and hence $X$ is a Cayley graph on $P L$. However, Lemma 3.2 implies that $L$ cannot be normal in $P L$, a contradiction.

Suppose now that the orbits of $N$ are of length 2 . Then $\left|X_{N}\right|=2 p$. By Proposition 2.2, $X_{N}$ is symmetric. If $X_{N}$ has valency $q$ then $X \cong X_{N}\left[2 K_{1}\right]$, which is symmetric, a contradiction. Thus, $X_{N}$ has valency $2 q$. In this case, $L_{u}=1$ and $L$ acts regularly on each orbit of $N$. It follows that $L=N \cong \mathbb{Z}_{2}$ and the quotient graph $X_{N}$ of $X$ relative to $N$ is $A / N$-half-arc-transitive. Note that $P N / N$ is a Sylow $p$-subgroup of $A / N$. By Proposition 2.2, $P N \unlhd A$ or $X_{N} \cong K_{p, p}-p K_{2}$ with $2 q=p-1$. For the former, $P \unlhd A$ because $|N|=2$. For the latter, $X_{N}$ is bipartite. Let $(A / N)^{*}$ be the subgroup of $A / N$ fixing the bipartite sets, say $\Sigma$ and $\Sigma^{\prime}$, of $X_{N}$ setwise. Since $X_{N} \cong K_{p, p}-p K_{2}$, $(A / N)^{*}$ acts faithfully on $\Sigma$ and $\Sigma^{\prime}$, respectively. Since $A / N$ is vertex-transitive on $X_{N}$, $\left|A / N:(A / N)^{*}\right|=2$. Assume $\Delta \in \Sigma$ and $\Delta^{\prime} \in \Sigma^{\prime}$ such that $\Delta$ is not adjacent to $\Delta^{\prime}$ in $X_{N}$, and let $(A / N)_{\Delta}$ and $(A / N)_{\Delta^{\prime}}$ be the stabilizers of $\Delta$ and $\Delta^{\prime}$ in $A / N$, respectively. Then $(A / N)_{\Delta}=(A / N)_{\Delta^{\prime}} \leq(A / N)^{*}$. By the half-arc-transitivity of $A / N$ on $X_{N}$, the stabilizer $(A / N)_{\Delta}$ cannot be transitive on $\Sigma^{\prime} \backslash\left\{\Delta^{\prime}\right\}$. It follows that $(A / N)^{*}$ is not 2transitive on $\Sigma$ and $\Sigma^{\prime}$. Since $|\Sigma|=\left|\Sigma^{\prime}\right|=p$, by Proposition 2.6, $(A / N)^{*}$ has a normal Sylow $p$-subgroup, which is also a normal Sylow $p$-subgroup of $A / N$ because $\mid A / N$ : $(A / N)^{*} \mid=2$. It follows that $P N \unlhd A$ and hence $P \unlhd A$.

Now assume that $A$ has no non-trivial normal 2-subgroups. Again we prove that $P \unlhd A$. Suppose that $A$ is primitive on $V(X)$. By Proposition 2.6, $A$ is 2-transitive on $V(X)$ provided $p \neq 7,13$ or 17 . Since $X$ is half-arc-transitive, $A$ is not 2-transitive on $V(X)$. It follows that $p=7,13$ or 17, which is impossible by Proposition 2.4. Thus, $A$ is imprimitive on $V(X)$. Let $B$ be a non-trivial block of $A$ on $V(X)$. Since $|V(X)|=4 p$, we have $|B|=2,4, p$ or $2 p$. It follows that $\mathcal{B}=\left\{B^{a} \mid a \in A\right\}$ is a complete block system of $A$ on $V(X)$. Consider the quotient graph $X_{\mathcal{B}}$ relative to $\mathcal{B}$ and let $K$ be the kernel of $A$ on $\mathcal{B}$. Then $A / K \leq \operatorname{Aut}\left(X_{\mathcal{B}}\right)$. Since $X$ is $A$-edge-transitive, $X_{\mathcal{B}}$ is $A / K$-edge-transitive. Let $B^{\prime} \in \mathcal{B}$ be adjacent to $B$ in $X_{\mathcal{B}}$ and let $k$ be the number of edges in $X$ between $B$ and $B^{\prime}$. Then $X_{\mathcal{B}}$ has valency $\frac{2 q|B|}{k}$. Assume $u \in B$ and choose $B^{\prime} \in \mathcal{B}$ such that $B^{\prime}$ contains an out-neighbor of $u$ in $\mathcal{O}$. Recall that $\alpha \in A_{u}$ and $\alpha$ permutes the elements in $N^{+}(u)$ cyclically. Then $\alpha$ fixes $B$ setwise. Since $\left|N^{+}(u)\right|=q$ is a prime, either $B^{\prime}$ contains exactly one out-neighbor of $u$ in $\mathcal{O}$ or $N^{+}(u) \subseteq B^{\prime}$. In particular, if $|B|=p$ or $2 p$ then $N^{+}(u) \subseteq B^{\prime}$ and $\alpha \in K$. If $|B|=2$ or 4 then $B^{\prime}$ contains exactly one out-neighbor of $u$ in $\mathcal{O}$. It follows $K_{u}=1$ because $K_{u}$ fixes each out-neighbor of $u$ in $\mathcal{O}$. Thus, $|K| \leq 4$ and since $|V(X)|=4 p$ and $A$ has no non-trivial normal 2-group, one has $K=1$, implying $A \leq \operatorname{Aut}\left(X_{\mathcal{B}}\right)$. Consider the four cases $|B|=4,2, p$ or $2 p$, respectively.

Case I: $|B|=4$.

In this case, $K=1$ and $A \leq \operatorname{Aut}\left(X_{\mathcal{B}}\right)$. Since $|\mathcal{B}|=p$, Proposition 2.6 implies that either $P \unlhd A$ or $A$ is 2 -transitive on $\mathcal{B}$. First suppose that $A$ is 2 -transitive on $\mathcal{B}$. Then $X_{\mathcal{B}} \cong K_{p}$ and $(p-1) k=4 \cdot 2 q=8 q$, where $k$ is the number of edges in $X$ between $B$ and $B^{\prime}$. It follows that $k=1,2$ or 4 . The 2-transitivity of $A$ on $\mathcal{B}$ implies that the number of directed edges in $\mathcal{O}$ with direction from $B$ to $B^{\prime}$ is equal to that of directed 
edges with direction from $B^{\prime}$ to $B$. Thus, half-arc-transitivity of $X$ implies that $k \neq 1$, and hence $k=2$ or 4 and $p-1=2 q$ or $4 q$. Again by Proposition 2.6, $\operatorname{soc}(A) \cong A_{p}$, $\operatorname{PSL}\left(2,2^{2^{s}}\right)$ with $p=2^{2^{s}}+1, \operatorname{PSL}(m, r)$ with $p=\frac{r^{m}-1}{r-1}, \operatorname{PSL}(2,11), M_{11}$ or $M_{23}$. If $\operatorname{soc}(A) \cong A_{p}$ then $|A|$ is divisible by $\frac{1}{2} p$ !. From elementary number theory it is well known that there exists a prime $t$ between $q$ and $2 q$. Since $p-1=2 q$ or $4 q$, one has $t|| A \mid$, which is impossible because $|A|=4 p q n$ where each prime divisor of $n$ is a divisor of $q$ !. If $\operatorname{soc}(A) \cong \operatorname{PSL}\left(2,2^{2^{s}}\right)$ then $p-1=2^{2^{s}} \neq 2 q$ or $4 q$, which is clearly impossible. If $\operatorname{soc}(A) \cong \operatorname{PSL}(m, r)$ then $p=\frac{r^{m}-1}{r-1}=r^{m-1}+\cdots+r+1$ and $m \geq 3$ is odd. It follows that $r(1+r) \mid(p-1)$, which is also impossible because $p-1=2 q$ or $4 q$ and $q \geq 5$ (note that $4 \cdot 5+1=21$ is not a prime). Thus, $\operatorname{soc}(A) \cong \operatorname{PSL}(2,11), M_{11}$ or $M_{23}$. It follows that $A \cong \operatorname{PSL}(2,11), \operatorname{PGL}(2,11), M_{11}$ or $M_{23}$ because $|\operatorname{Out}(\operatorname{PSL}(2,11))|=2$, and $\left|\operatorname{Out}\left(M_{11}\right)\right|=\left|\operatorname{Out}\left(M_{23}\right)\right|=1$ (see [8]). Note that $|\operatorname{PSL}(2,11)|=2^{2} \cdot 3 \cdot 5 \cdot 11$, $\left|M_{11}\right|=2^{4} \cdot 3^{2} \cdot 5 \cdot 11$ and $\left|M_{23}\right|=2^{7} \cdot 3^{2} \cdot 5 \cdot 7 \cdot 11 \cdot 23$. Since $|V(X)|=4 p$, if $A \cong \operatorname{PSL}(2,11)$ or $\operatorname{PGL}(2,11)$ then $A_{u}$ is a subgroup of $A$ of order 15 or 30 , respectively, which is not true by [8]. Similarly, $A \neq M_{11}$ or $M_{23}$ because $M_{11}$ and $M_{23}$ have no subgroups of order $2^{2} \cdot 3^{2} \cdot 5$ and $2^{5} \cdot 3^{2} \cdot 5 \cdot 7 \cdot 11$, respectively. Thus, $P \unlhd A$.

Case II: $|B|=2$.

In this case $K=1$ and $A \leq \operatorname{Aut}\left(X_{\mathcal{B}}\right)$. By Proposition 2.2, $X_{\mathcal{B}}$ is symmetric. Let $B=\{u, v\}$ and $B^{\prime}=\left\{u^{\prime}, v^{\prime}\right\}$, and assume that $\left(u, u^{\prime}\right)$ is a directed edge in $\mathcal{O}$. Suppose that $u$ has two neighbors in $X$ in $B^{\prime}$. Since $B^{\prime}$ contains exactly one out-neighbor of $u$ in $\mathcal{O}$, $\left(v^{\prime}, u\right)$ is a directed edge in $\mathcal{O}$. Since $A$ is transitive on the directed edges in $\mathcal{O}, A$ contains an element mapping $\left(u, u^{\prime}\right)$ to $\left(v^{\prime}, u\right)$, forcing that $k \geq 3$. Recall that $X_{\mathcal{B}}$ has valency $\frac{2 q|B|}{k}$. It follows that $k=4$ and hence $X=X_{\mathcal{B}}\left[2 K_{1}\right]$, which is symmetric, a contradiction. Thus, there are exactly 2 or 1 edges in $X$ between $B$ and $B^{\prime}$, meaning that $X_{\mathcal{B}}$ has valency $2 q$ or $4 q$. Recall that $A$ has no non-trivial normal 2-subgroup and then, by Proposition 2.2, $P \unlhd A$, or $X_{\mathcal{B}} \cong K_{p, p}-p K_{2}$ with $p-1=2 q$ or $4 q$, or $X_{\mathcal{B}} \cong G(2, p, 2 q)$ with $p-1=2 q$.

Assume that $X_{\mathcal{B}} \cong K_{p, p}-p K_{2}$. Let $\mathcal{B}_{1}$ and $\mathcal{B}_{2}$ be the bipartite sets of $X_{\mathcal{B}}$ such that $B \in \mathcal{B}_{1}$ and $B^{\prime} \in \mathcal{B}_{2}$, and let $A^{*}$ be the subgroup of $A$ fixing $\mathcal{B}_{1}$ and $\mathcal{B}_{2}$ setwise. Then $\left|A: A^{*}\right|=2$ and $A^{*} \unlhd A$. There is a unique block $C \in \mathcal{B}_{2}$ which is not adjacent to $B$ in $X_{\mathcal{B}}$. Let $A_{B}$ and $A_{C}$ be the block stabilizers of $B$ and $C$ in $A$, that is the subgroups of $A$ fixing $B$ and $C$, respectively. Then $A_{B}=A_{C} \leq A^{*}$ and $A^{*}$ is faithful on $\mathcal{B}_{1}$ and $\mathcal{B}_{2}$. Clearly, $P \leq A^{*}$. By Proposition 2.6, $P \unlhd A^{*}$ or $A^{*}$ is 2-transitive on $\mathcal{B}_{1}$ and $\mathcal{B}_{2}$.

Suppose that $A^{*}$ is 2 -transitive on $\mathcal{B}_{1}$ and $\mathcal{B}_{2}$. Then $A_{B}=A_{C}$ is transitive on $\mathcal{B}_{1} \backslash\{B\}$ and $\mathcal{B}_{2} \backslash\{C\}$, respectively. Note that $p-1=4 q$ or $2 q$. If $p-1=4 q$ then there is exactly one directed edge in $\mathcal{O}$ between $B$ and $B^{\prime}$. Since $X$ is half-arc-transitive, $A_{B}$ has two orbits on $\mathcal{B}_{2} \backslash\{C\}$, a contradiction. Thus, $p-1=2 q$ and there are exactly two directed edges in $\mathcal{O}$ between $B$ and $B^{\prime}$. These two directed edges have different direction, that is one from $B$ to $B^{\prime}$ and the other from $B^{\prime}$ to $B$, because $A_{B}$ is transitive on $\mathcal{B}_{2} \backslash\{C\}$. This means that the permutation $\beta$ on $V(X)$ interchanging the two vertices in each block of $\mathcal{B}$ cannot be an automorphism of $\mathcal{O}$. On the other hand, since the induced subgraph $\left\langle B \cup B^{\prime}\right\rangle$ of $B \cup B^{\prime}$ in $X$ is a matching, one has $\beta \in A$, contrary to the fact that $A \leq \operatorname{Aut}(\mathcal{O})$. Thus, $P \unlhd A^{*}$ and hence $P \unlhd A$.

Assume that $X_{\mathcal{B}} \cong G(2, p, 2 q)$ with $p-1=2 q$. By Proposition $2.2, \operatorname{Aut}\left(X_{\mathcal{B}}\right) \cong \mathbb{Z}_{2}^{p} \rtimes$ $S_{p}$. Thus, $\operatorname{Aut}\left(X_{\mathcal{B}}\right)$ has a normal subgroup $N$ such that $N \cong \mathbb{Z}_{2}^{p}$. Since $\left|V\left(X_{\mathcal{B}}\right)\right|=2 p$, the orbits of $N$ have size 2 , which are blocks of $\operatorname{Aut}\left(X_{\mathcal{B}}\right)$ (in fact $X_{\mathcal{B}} \cong K_{p}\left[2 K_{1}\right]$ ). It follows that $A$ has blocks of size 4 on $V(X)$. By Case I, $P \unlhd A$. 
Case III: $|B|=p$.

In this case, $N^{+}(u) \subseteq B^{\prime}$ and $\alpha \in K$, where $\alpha \in A_{u}$ permutes the elements in $N^{+}(u)$ cyclically. Since $\left|V\left(X_{\mathcal{B}}\right)\right|=4$, any element of order $p$ in $A$ fixes each vertex of $X_{\mathcal{B}}$. Thus, $p q|| K \mid$ and $X_{\mathcal{B}}$ is a 4-cycle, say $V\left(X_{\mathcal{B}}\right)=\left(B_{0}, B_{1}, B_{2}, B_{3}\right)$, where $B_{i}$ is adjacent to $B_{i+1}$ for each $i \in \mathbb{Z}_{4}$. Let $Y=\left\langle B_{0} \cup B_{1}\right\rangle$ be the subgraph induced by $B_{0} \cup B_{1}$ in $X$. Then $Y$ is a $q$-valent edge-transitive graph of order $2 p$, and all edges in $Y$ have the same direction either from $B_{0}$ to $B_{1}$ or from $B_{1}$ to $B_{0}$ in $\mathcal{O}$, forcing $A / K \cong \mathbb{Z}_{4}$. If $Y \cong K_{p, p}$ then $X \cong C_{4}\left[p K_{1}\right]$, which is symmetric, a contradiction. One may thus assume that $Y \nRightarrow K_{p, p}$. If $K$ is unfaithful on $B_{0}$ then the kernel of $K$ on $B_{0}$ is transitive on $B_{1}$ because $\left|B_{1}\right|=p$, forcing that $Y \cong K_{p, p}$, a contradiction. Thus, $K \leq \operatorname{Aut}(Y)$. By Proposition 2.2, either $Y \cong G(2 p, q)$ with $q \mid(p-1)$ and $(p, q) \neq(11,5)$, and $\operatorname{Aut}(Y) \cong\left(\mathbb{Z}_{p} \rtimes \mathbb{Z}_{q}\right) \rtimes \mathbb{Z}_{2}$, or $Y \cong G(2 \cdot 11,5)$ and $\operatorname{Aut}(Y) \cong \operatorname{PSL}(2,11) \rtimes \mathbb{Z}_{2}$. For the former, since $K$ fixes $B_{0}$ and $B_{1}$ setwise and $p q|| K \mid$, one has $|K|=p q$. For the latter, $Y \cong G(2 \cdot 11,5)$ and $\operatorname{Aut}(Y) \cong$ $\operatorname{PSL}(2,11) \rtimes \mathbb{Z}_{2}$. Since $K$ fixes $B_{0}$ and $B_{1}$ setwise, $K \lesssim \operatorname{PSL}(2,11)$. Suppose that $K \cong$ $\operatorname{PSL}(2,11)$. Let $C_{A}(K)$ be the centralizer of $K$ in $A$. Then $C_{A}(K) \cap K=1$ because $K$ is non-abelian simple. Since $C_{A}(K) \cong C_{A}(K) /\left(K \cap C_{A}(K)\right) \cong K C_{A}(K) / K \leq A / K \cong$ $\mathbb{Z}_{4}$ and $A$ has no non-trivial normal 2-subgroup, one has $C_{A}(K)=1$. It follows that $A=A / C_{A}(K) \lesssim \operatorname{Aut}(\operatorname{PSL}(2,11)) \cong \operatorname{PGL}(2,11)$, contrary to the fact that $A / K \cong \mathbb{Z}_{4}$. This implies that $K$ is isomorphic to a proper subgroup of $\operatorname{PSL}(2,11)$ of order divisible by 55. By [8], $|K|=55$. Thus, we always have $|K|=p q$. Since $|A|=4 p q, P \unlhd K$ and hence $P \unlhd A$.

Case IV: $|B|=2 p$.

In this case, $X$ is a bipartite graph with bipartite sets $B$ and $B^{\prime}$. If $p=q$, then $X \cong$ $K_{2 p, 2 p}$ is symmetric, a contradiction. Thus $q<p$. Let $A^{*}$ be the subgroup of $A$ fixing $B$ and $B^{\prime}$ setwise. Then $\left|A: A^{*}\right|=2$ and $A^{*} \unlhd A$. Suppose that $A^{*}$ is 2 -transitive on $B$. Then every vertex in $B \backslash\{u\}$ has the same number of in-neighbors and the same number of outneighbors in $N^{+}(u)$ in $\mathcal{O}$, say $m_{1}$ and $m_{2}$, respectively. It follows that $q^{2}=(2 p-1) m_{1}$ and $q(q-1)=(2 p-1) m_{2}$, forcing $m_{1}-m_{2}=1$ and $q=2 p-1$, contrary to the fact that $q<p$. Since $|B|=2 p$, by Proposition $2.6 A^{*}$ is imprimitive on $B$. Note that $A^{*}$ is the block stabilizer of $B$ in $A$. Thus, every non-trivial block of $A^{*}$ on $B$ is also a block of $A$, which has size 2 or $p$. By Case II and Case III, $P \unlhd A$.

Now we are ready to prove the lemma. Since $P \unlhd A$, the orbits of $P$ are blocks of $A$ of length $p$. By the proof in Case III, $|A|=4 p q$ and hence $A$ is solvable. Clearly, the Hall $\{2, p\}$-subgroup of $A$ acts regularly on $V(X)$, implying that $X$ is a Cayley graph. By Lemma 3.2, one may let $X=\operatorname{Cay}(G, S)$, where $G=\left\langle a, b \mid a^{p}=b^{4}=1, b^{-1} a b=a^{r}\right\rangle$ with $r^{2} \equiv-1(\bmod p)$. Thus, $4 q \mid(p-1)$.

Since $P \unlhd A$, one has $P \leq R(G)$. Set $C=C_{A}(P)$. Since $R(G)$ is a Hall $\{2, p\}$ subgroup of $A$ and there is no involution in $G$ commuting with $a, C$ is a $p$-group or a $\{q, p\}$-group. If $q|| C \mid$ then $|C|=p q$ and $C$ has a normal Sylow $q$-subgroup, which is normal in $A$ because $C \unlhd A$. This implies that $A_{u} \unlhd A$, forcing $A_{u}=1$, a contradiction. It follows that $P=C$, and since $A / C=A / P \lesssim \operatorname{Aut}(P) \cong \mathbb{Z}_{p-1}$, one has $R(G) / P \unlhd A / P$, that is $R(G) \unlhd A$. Thus $X=\operatorname{Cay}(G, S)$ is a normal Cayley graph. By Proposition 2.1, $A=R(G) \rtimes \operatorname{Aut}(G, S)$ and since $|A|=4 p q$, there is an element $\delta$ of order $q$ in $\operatorname{Aut}(G, S)$.

The connectivity of $X$ implies $\langle S\rangle=G$. Since $G$ has a unique normal subgroup of order $2 p, S$ contains elements of order 4 . Each element of order 4 in $G$ is of the form $a^{i} b$ or $a^{i} b^{-1}$ for some integer $i$. It is easy to see that $\operatorname{Aut}(G)$ is transitive on the set $\left\{a^{i} b \mid 0 \leq i \leq\right.$ 
$p-1\}$. Thus, one may assume $b \in S$. Since there is no automorphism of $G$ mapping $b$ to $b^{-1}$, one may further assume $a^{\delta}=a^{s}$ and $b^{\delta}=a^{k} b(s \neq 0(\bmod p))$, and since $S^{\delta}=S$, we have that $E=\left\{b, b^{\delta}, \ldots, b^{\delta^{q-1}}\right\}=\left\{b, a^{k s[1]} b, a^{k s[2]} b, a^{k s[3]} b, \ldots, a^{k s[q-1]} b\right\}$ is a subset of $S$, where $s[i]=s^{i-1}+\cdots+s+1$ for $1 \leq i \leq q-1$ and $s[q]=s^{q-1}+\cdots+s+1=$ $0(\bmod p)$. It follows that $S=E \cup E^{-1}$ because $S=S^{-1}$. Clearly, $k \neq 0(\bmod p)$. Since the map $a \mapsto a^{k}, b \mapsto b$, induces an automorphism of $G$, we have $X \cong \mathcal{C}_{4 p}^{2 q}$.

\section{Acknowledgements}

This work was supported by the National Natural Science Foundation of China (10871021, 10911140266), the Fundamental Research Funds for the Central Universities (2009YJS036) and the Doctorate Foundation of Beijing Jiaotong University (141109522).

\section{References}

[1] B. Alspach, D. Marušič, L. Nowitz, Constructing graphs which are 1/2-transitive, J. Austral. Math. Soc. A 56 (1994), 391-402.

[2] B. Alspach, M. Y. Xu, 1/2-transitive graphs of order 3p, J. Algebraic Combin. 3 (1994), 347355.

[3] N. Biggs, Algebraic Graph Theory, second edn., Cambridge University Press, Cambridge, 1993.

[4] I. Z. Bouwer, Vertex and edge-transitive but not 1-transitive graphs, Canad. Math. Bull. 13 (1970), 231-237.

[5] C. Y. Chao, On the classification of symmetric graphs with a prime number of vertices, Trans. Amer. Math. Soc. 158 (1971), 247-256.

[6] Y. Cheng, J. Oxley, On weakly symmetric graphs of order twice a prime, J. Combin. Theory B 42 (1987), 196-211.

[7] M. D. E. Conder, D. Marušič, A tetravalent half-arc-transitive graph with non-abelian vertex stabilizer, J. Combin. Theory B $\mathbf{8 8}$ (2003), 67-76.

[8] H. J. Conway, R. T. Curtis, S. P. Norton, R. A. Parker, R. A Wilson, Atlas of Finite Groups, Oxford University Press, Oxford, 1985.

[9] J. D. Dixon, B. Mortimer, Permutation Groups, Springer-Verlag, New York, 1996.

[10] S. F. Du, M. Y. Xu, Vertex-primitive 1/2-arc-transitive graphs of smallest order, Comm. Algebra 27 (1999), 163-171.

[11] X. G. Fang, C. H. Li, M. Y. Xu, On edge-transitive Cayley graphs of valency 4, European J. Combin. 25 (2004), 1107-1116.

[12] Y.-Q. Feng, J. H. Kwak, M. Y. Xu, J.-X. Zhou, Tetravalent half-arc-transitive graphs of order $p^{4}$, European J. Combin. 29 (2008), 555-567.

[13] Y.-Q. Feng, K. S. Wang, C. X. Zhou, Tetravalent half-transitive graphs of order $4 p$, European J. Combin. 28 (2007), 726-733.

[14] Y.-Q. Feng, J. H. Kwak, C. X. Zhou, Constructing even radius tightly attached half-arctransitive graphs of valency four, J. Algebraic Combin. 26 (2007), 431-451.

[15] D. F. Holt, A graph which is edge-transitive but not arc transitive, J. Graph Theory 5 (1981), 201-204.

[16] J. H. Kwak, Y. S. Kwon, J. M. Oh, Infinitely many one-regular Cayley graphs on dihedral groups of any prescribed valency, J. Combin. Theory B 98 (2008), 585-598. 
[17] C. H. Li, Z. P. Lu, D. Marušič, On primitive permutation groups with small suborbits and their orbital graphs, J. Algebra 279 (2004), 749-770.

[18] C. H. Li, H. S. Sim, On half-transitive metacirculant graphs of prime-power order, J. Combin. Theory B 81 (2001), 45-57.

[19] H. L. Li, J. Wang, L. Y. Wang, M. Y. Xu, Vertex primitive graphs of order containing a large prime factor, Comm. in Algebra 22 (1994) 3449-3477.

[20] M. W. Liebeck, J. Saxl, Primitive permutation groups containing an element of large prime order, J. London. Math. Soc. 31 (1985), 237-249.

[21] A. Malnič, D. Marušič, Constructing 4-valent 1/2-transitive graphs with a nonsolvable automorphism group, J. Combin. Theory B 75 (1999), 46-55.

[22] A. Malnič, D. Marušič, Constructing 1/2-arc-transitive graphs of valency 4 and vertex stabilizer $\mathbb{Z}_{2} \times \mathbb{Z}_{2}$, Discrete Math. 245 (2002), 203-216.

[23] D. Marušič, Half-transitive group actions on finite graphs of valency $4, J$. Combin. Theory $B$ 73 (1998), 41-76.

[24] D. Marušič, Quartic half-transitive graphs with large vertex stabilizers, Discrete Math. 299 (2005), 180-193.

[25] D. Marušič, R. Nedela, Finite graphs of valency 4 and girth 4 admitting half-transitive group actions, J. Austral. Math. Soc. 73(2002) 155-170.

[26] D. Marušič, R. Nedela, Partial line graph operator and half-arc-transitive group actions, Math. Slovaca 51 (2001), 241-257.

[27] D. Marušič, C. E. Praeger, Tetravalent graphs admitting half-transitive group action: alternating cycles, J. Combin. Theory B 75 (1999), 188-205.

[28] D. Marušič, P. Šparl, On quartic half-arc-transitive metacirculants, J. Algebraic Combin. 28 (2008), 365-395.

[29] D. Marušič, A. Waller, Half-transitive graphs of valency 4 with prescribed attachment numbers, J. Graph Theory 34 (2000), 89-99.

[30] D. Marušič, M. Y. Xu, A $\frac{1}{2}$-transitive graph of valency 4 with a nonsolvable group of automorphisms, J. Graph Theory 25 (1997), 133-138.

[31] C. E. Praeger, M. Y. Xu, Vertex primitive graphs of order a product of two distinct primes, $J$. Combin. Theory B 59 (1993), 245-266.

[32] P. Šparl, A classification of tightly attached half-arc-transitive graphs of valency 4, J. Combin. Theory B 98 (2008), 1076-1108.

[33] D. E. Taylor, M.Y. Xu, Vertex-primitive 1/2-transitive graphs, J. Austral. Math. Soc. A 57 (1994), 113-124.

[34] W. T. Tutte, Connectivity in Graphs, University of Toronto Press, Toronto, 1966.

[35] R. J. Wang, Half-transitive graphs of order a product of two distinct primes, Comm. Algebra 22 (1994), 915-927.

[36] R. J. Wang, M. Y. Xu, A classification of symmetric graphs of order $3 p$, J. Combin. Theory B 58 (1993), 197-216.

[37] X. Y. Wang, Y.-Q. Feng, Hexavalent half-arc-transitive graphs of order $4 p$, European J. combin. 30 (2009), 1263-1270.

[38] M. Y. Xu, Half-transitive graphs of prime-cube order, J. Algebraic Combin. 1 (1992), 275-282.

[39] C. X. Zhou, Y.-Q. Feng, An infinite family of tetravalent half-arc-transitive graphs, Discrete Math. 306 (2006), 2205-2211. 